\title{
ESTUDO DO EFEITO DE CICLOS DE MOLHAGEM E SECAGEM NA DURABILIDADE DE ARGAMASSAS COM ADIÇÃO DE RESÍDUO DA PRODUÇÃO DE PAPEL*
}

\author{
Afonso Rangel Garcez de Azevedo ${ }^{1 *}$ \\ Jonas Alexandre ${ }^{2}$ \\ Euzébio Bernabé Zanelato ${ }^{3}$ \\ Markssuel Teixeira Marvila ${ }^{4}$ \\ Sergio Neves Monteiro ${ }^{5}$ \\ Gustavo de Castro Xavier ${ }^{6}$ \\ Maria Lúcia Pereira Antunes ${ }^{7}$ \\ Welington Fernandes Junior ${ }^{8}$ \\ Bruno Rangel França ${ }^{9}$
}

\section{Resumo}

A produção de papel gera grande quantidade de resíduo em diferentes etapas da produção, constituído basicamente de celulose e caulim, que atualmente não tem um destino adequado e acaba gerando custos para indústria. O objetivo deste trabalho é avaliar o efeito de ciclos de molhagem e secagem na substituição deste resíduo em massa de cal (5, 10 e 15\%) em argamassas a base de cimento e cal, traço 1:1:6 (cimento: cal: areia), para assentamento de blocos e revestimento de paredes e tetos onde serão analisadas propriedades como a perda de massa e resistência mecânica após a ocorrência de ciclos de imersão em água a temperatura ambiente e secagem em estufa em períodos de 15, 30 e 45 dias após o tempo de cura (28 dias). Os resultados encontrados mostraram que a perda de massa apresentada não é significativa, sendo maior em 15\%, provavelmente devido à tendência de preenchimento dos poros na matriz cimentícia, quando correlacionados com resultados da argamassa no estado fresco. Quanto à resistência mecânica a compressão, as argamassas não apresentaram perdas significativas que impedisse seu uso em todos os ciclos analisados. Pode-se concluir que os traços estudados apresentam desempenho satisfatório em ensaios de ciclagem podendo com uso recomendado.

Palavras-chave: Resíduo; Durabilidade; Argamassa.

\section{Abstract \\ COST ANALYSIS USING NON-STANDARDIZED CERAMIC BLOCKS}

Paper production generates a large amount of waste at different stages of production, basically made up of cellulose and kaolin, which currently does not have an adequate destination and ends up generating costs for industry. The purpose of this work is to evaluate the effect of wetting and drying cycles on the substitution of lime and cement based mortar with lime (1, 1 and 6) in lime mass (5, 10 and 15\%), Sand), for block set-ting and wall and ceiling coating, where properties such as mass loss and mechanical resistance will be analyzed after immersion cycles in room temperature water and oven drying in periods of 15, 30 and 45 days After the cure time (28 days). The results showed that the loss of mass presented is not significant, being the largest in 15\%, probably due to the tendency of pore filling in the cement matrix, when correlated with the results of the mortar in the fresh state. As for the mechanical resistance to compression, the mortars did not present significant losses that prevented their use in all cycles analyzed. It can be concluded that the traits studied present satisfactory performance in cycling trials and may have its recommended use.

Keywords: Waste; Durability; Mortar. 
1 Engenheiro Civil, Mestre em Engenharia Civil, Laboratório de Engenharia Civil, Universidade Estadual do Norte Fluminense, Campos dos Goytacazes, Rio de Janeiro, Brasil.

2 Engenheiro Civil, Doutor em Ciências de Engenharia, Laboratório de Engenharia Civil, Universidade Estadual do Norte Fluminense, Campos dos Goytacazes, Rio de Janeiro, Brasil.

3 Engenheiro Civil, Mestre em Engenharia Civil, Laboratório de Engenharia Civil, Universidade Estadual do Norte Fluminense, Campos dos Goytacazes, Rio de Janeiro, Brasil.

4 Engenheiro Civil, Graduado em Engenharia Civil, Laboratório de Engenharia Civil, Universidade Estadual do Norte Fluminense, Campos dos Goytacazes, Rio de Janeiro, Brasil.

5 Engenheiro de Metalurgia e Materiais, Doutor em Metalurgia e Materiais, Instituto Militar de Engenharia, Rio de Janeiro, Rio de Janeiro, Brasi

6 Engenheiro Civil, Doutor em Ciências de Engenharia, Laboratório de Engenharia Civil, Universidade Estadual do Norte Fluminense, Campos dos Goytacazes, Rio de Janeiro, Brasil

7 Física, Doutora em Física, Universidade Estadual Paulista Júlio de Mesquita Filho, São Paulo, Brasil

8 Engenheiro Civil, Graduado em Engenharia Civil, Laboratório de Engenharia Civil, Universidade Estadual do Norte Fluminense, Campos dos Goytacazes, Rio de Janeiro, Brasil

9 Engenheiro Civil, Graduado em Engenharia Civil, Laboratório de Engenharia Civil, Universidade Estadual do Norte Fluminense, Campos dos Goytacazes, Rio de Janeiro, Brasil. 


\section{INTRODUÇÃO}

A regulamentação da Politica Nacional de Resíduos Sólidos trouxe avanços significativos na área de resíduos sólidos [1]. Neste contexto a indústria de papel e celulose gera em torno de $35 \%$ de resíduos variados em comparação com o volume de papel produzido [2]. O resíduo da indústria de papel é constituído basicamente de caulim e celulose e outros materiais em menores proporções [3], tem sua consistência similar a uma lama extremamente úmida (65 a 70\%) [4] que é alocada em Estações de Tratamento de Efluentes dentro das plantas industriais. O Brasil destaca-se no cenário mundial de produção de papel, sendo segundo dados da Associação Brasileira de Papel e Celulose [5] a produção de papel só vem aumento. Assim com o incremento da produção, as indústrias passaram a enfrentar de maneira mais impactante a questão do gerenciamento de seus resíduos sólidos, já que para atenderem a legislação ambiental vigente necessitam pagar para a disposição do material em aterros sanitários [6]. A possibilidade de incorporar resíduos sólidos em materiais de construção não é algo novo e existem diversas estudos neste segmento, principalmente em materiais cerâmicos, como a incorporação deste lodo em cerâmica vermelha [7], e cimentícios, incorporação em blocos de cimento e argamassas [8]. A durabilidade é susceptibilidade dos materiais a alteração ao longo de sua vida útil [9], que pode ser de maneira natural (no campo) ou acelerada (em laboratório). Os ensaios de ciclagem mostram o comportamento do material durante ciclos contínuos de molhagem e secagem. No caso de argamassas usadas para revestimento que estão submetidas a constantes ciclos em sua utilização na construção civil e o conhecimento do seu comportamento nesta situação mostra-se extremamente importante. O objetivo deste trabalho foi a avaliação do processo de degradação de argamassas com traço 1:1:6 (cimento: cal: areia) em diferentes níveis de substituição da cal pelo resíduo da indústria de papel $(5,10,15$ e $20 \%)$ quando expostos a ciclos de molhagem e secagem onde serão avaliados os parâmetros de perda de massa e resistência mecânica à compressão.

\section{MATERIAIS E MÉTODOS}

O resíduo utilizado neste trabalho foi fornecido por uma industrial de papel localizada no Noroeste Fluminense, onde a mesma ficou responsável pela coleta e transporte do resíduo até a Universidade Estadual do Norte Fluminense. Ao chegar o material foi identificado e acondicionado para se iniciar a secagem e homogeneização, devido ao elevado teor de umidade o mesmo passou por um processo de secagem natural e outro artificial em estufa microprocessada a temperatura de $110^{\circ} \mathrm{C}$ ao longo das primeiras 24 horas, após esse tempo foi feito um monitoramento até que sua massa fique constante, ou seja esteja seco para iniciar o processo de homogeneização. O processo de homogeneização consistiu na moagem em moinho de bolas de 30 rotações por minuto. O respectivo moinho é de modelo tambor com carga moedora de 12 bolas de aço (cada uma com aproximadamente $48 \mathrm{~mm}$ de diâmetro e massa de $390 \mathrm{~g}$ a $445 \mathrm{~g}$ ) atende aos requisitos da NBR NM 51: 2001 [10]. Após este processo o resíduo encontra-se em condições de incorporação na argamassa. A areia utilizada para este trabalho é proveniente da bacia sedimentar do rio Paraíba do Sul. O cimento e a cal utilizada são comercias do tipo CPII e CHIII respectivamente.

O processo de confecção da argamassa a base de cimento e cal atendeu aos requisitos da norma brasileira [11], assim como a moldagem de seus corpos de 
prova prismáticos. O procedimento do ensaio de ciclagem constituiu em ciclos onde a argamassa será imersa em água durante um período de tempo a uma temperatura de $23^{\circ} \mathrm{C}$ e na sequência colocada em estufa, a uma temperatura de $100^{\circ} \mathrm{C}$, com intervalos de 30 minutos [12]. As argamassas serão curadas inicialmente em condições ambientes do laboratório durante 28 dias e submetidas três padrões de ciclagem, o primeiro ciclo tem duração de 15 dias além do tempo de cura, o segundo têm 30 dias além a cura e o terceiro 45 dias a mais. A cada dia os corpos de prova ficam expostos à ciclagem por mais 14 dias, totalizando 7 ciclos. Cada dia corresponde a 13 horas do corpo de prova submerso em água a temperatura ambiente, 30 minutos em temperatura ambiente, e mais 10 horas em estufa a $100^{\circ} \mathrm{C}$, após sua retirada mais 30 minutos ao ambiente até iniciar novamente uma etapa do ciclo finalizando nos dias de cada tipo [1, 2 ou 3].

\section{RESULTADOS E DISCUSSÃO}

Os resultados referentes à perda de massa das argamassas a base de cimento e cal, em diferentes níveis de incorporação, são mostrados na Tabela 1, que segue abaixo.

Tabela 1 - Perda de massa em gramas em diferentes níveis de incorporação e ciclos.

\begin{tabular}{|c|c|c|c|}
\hline Nível de Incorporação & Ciclo 1 & Ciclo 2 & Ciclo 3 \\
\hline $0 \%$ & 6,60 & 5,40 & 8,20 \\
\hline $5 \%$ & 8,00 & 6,60 & 7,26 \\
\hline $10 \%$ & 8,70 & 4,43 & 9,56 \\
\hline $15 \%$ & 8,55 & 9,30 & 12,10 \\
\hline $20 \%$ & 9,26 & 10,80 & 18,53 \\
\hline
\end{tabular}

A Figura 1 mostra a resistência mecânica das argamassas ao estado natural em seus níveis de incorporação e após a passagem pelos ciclos de molhagem e secagem.

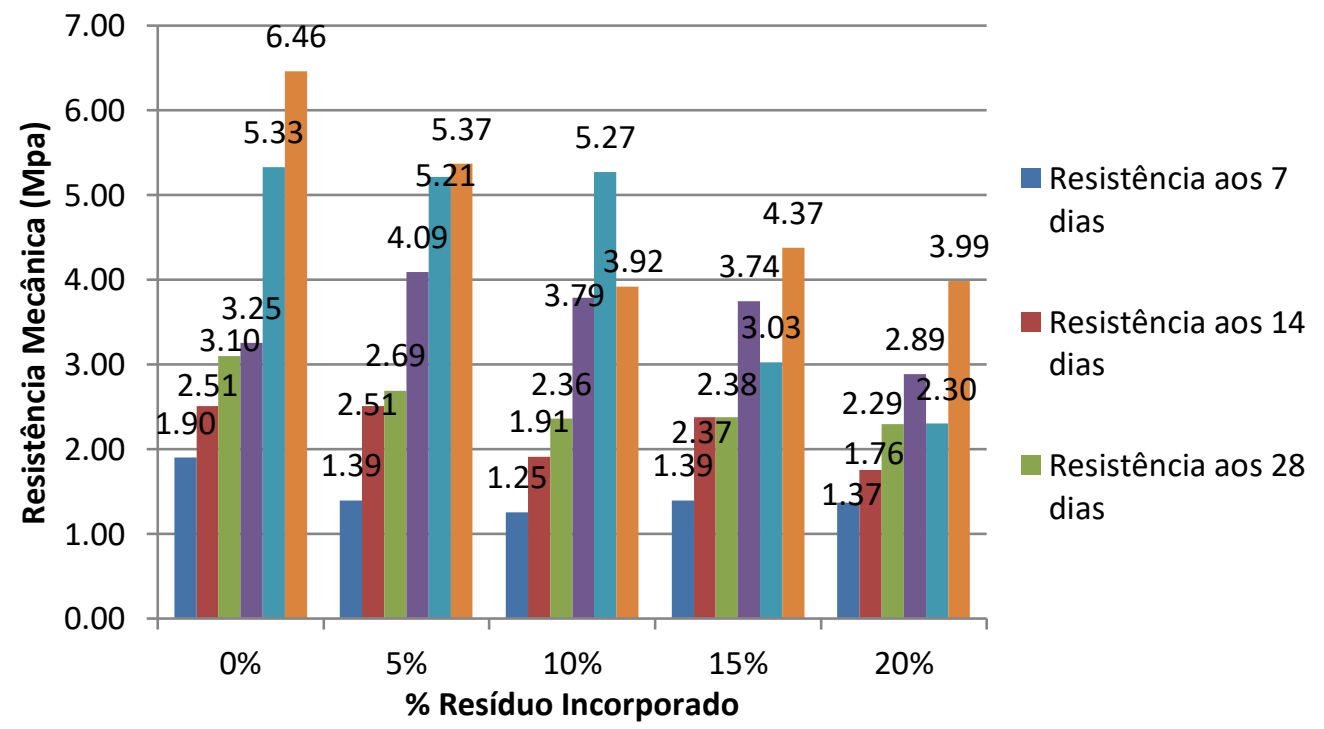

Figura 1 - Resistência mecânica (Mpa) nos diferentes níveis de incorporação e ciclos estudados. Fonte: autoria própria 
Os dados da Tabela 1 nos mostram que o nível de incorporação que mais apresentou perda de massa foi o de $20 \%$, este fato deve-se ao comportamento que o resíduo desempenha dentro da matriz, o coeficiente de capilaridade ${ }^{(6)}$ das argamassas com este nível de incorporação é maior que os demais níveis propiciando uma maior passagem de água pelos poros afetando a integridade da argamassa no estado endurecido.

Já a Figura 1 nos mostra que a tendência e de queda da resistência à compressão à medida que o nível de incorporação aumenta. Já os resultados dos ciclos somente não tem relação linear de crescimento ou decrescimento mostrando uma instabilidade do comportamento do material frente a sua compacidade, que pode afetar sua resistência. Há estudos relativos ao comportamento que este resíduo pode desenvolver junto ao cimento, inclusive analisando sua atividade pozolanica [13], onde indica que o mesmo pode ser pozolanico, o que poderia influenciar em seu comportamento mecânico.

\section{CONCLUSÃo}

Com os resultados aferidos neste estudo pode influir que em todos os níveis de incorporação analisados as argamassas desempenham um papel satisfatório, entretanto em elevadas concentrações o material pode desencadear em perda de propriedades mecânicas. Como a problema de disposição de resíduos sólidos industriais passa pela quantidade de material que é gerado e quantidade do produto no qual é incorporado, e considerando que argamassas são produtos de grande consumo, sua incorporação em pequenos níveis como 5\% a 10\% tem pouca alteração das propriedades iniciais e resolvem a questão de disposição do material, tornando-se uma alternativa viável economicamente e tecnicamente.

\section{REFERÊNCIAS}

1. LEVY, S. M.; HELENE, P. Durability of recycled aggregates concrete: a safe way to sustainable development. Cement and Concrete Research, v 34, p. 1975-1980, 2004.

2. NOLASCO, A.M. Utilização de resíduos da indústria de papel na produção de materiais para a construção civil. 1993. 140f. Dissertação (Mestrado) Universidade de São Paulo - Escola de Engenharia de São Carlos, São Carlos, 1993.

3. Zanella, B.P., E.B. Sá, N.O. Acorinti, I.C.B. Trannin e S.J.C. Simões. DURABILITY OF MIXED MORTAR LINING CONTAINING DREGS-GRITS. American Journal of Environmental Science. V.10, p. 44-47, 2014.

4.VAZ, A.R.; VALENTE, C.A. Lama do efluente de fábrica de celulose - algumas soluções para seu aproveitamento. In: 22 Congresso Anual de Celulose e Papel. Anais, São Paulo, 1989. Vol. 1p.625-641.

5. BRACELPA - Associação Brasileira de Celulose. Publicação mensal de março de 2014 . Disponível em: < http://bracelpa.org.br > Acesso em 29 mar. 2017.

6. AZEVEDO, A.R.G. Avaliação da Incorporação do Lodo Primário da ETE da Indústria de Papel Reciclado na Produção de Argamassas. 2015. $102 f$. Dissertação (Mestrado). Universidade Estadual do Norte Fluminense Darcy Ribeiro, Campos dos Goytacazes. 2015. 
7.VIEIRA, C.M.F.; PINHEIRO, R.M.; RODRIGUEZ, R.J.S.; CANDIDO, V.S. Clay bricks added with effluent sludge from paper industry: Technical, economical and environmental benefits. Applied Clay Science. V.4 , p. 134 - 141, 2016.

8.KARAHAN, O; ATIS, C.D. The durability properties of polypropylene fiber reinforced fly ash concrete. Materials Design, v. 32, p. 1044-1049, 2011

9. APONTE, D.F.; BARRA, M.; VÁZQUEZ, E. Durability and cementing efficiency of fly ash in concretes. Construction and Building Materials. V. 30, P. 537-546, 2012.

10. ASSOCIAÇÃO BRASILEIRA DE NORMAS TÉCNICAS. NBR NM51: Agregado graúdo - Ensaio de abrasão "Los Ángeles". Rio de janeiro, 2001.

11.ASSOCIAÇÃO BRASILEIRA DE NORMAS TÉCNICAS. NBR 13276: Argamassas para assentamento e revestimento de paredes e tetos - Preparo da mistura e determinação do índice de consistência. Rio de janeiro, 2005.

12.FARIA FILHO, J.; MENEZES, R. R.;FERREIRA, H. S.; SANTANAL, L. N. L.; NEVES, G. A.; FERREIRA, H. C. Estudo da durabilidade de argamassas alternativas contendo resíduos. Cerâmica. v. 57.p. 123 - 131. 2011

13. AZEVEDO, A. R. G.; ALEXANDRE, J. ; PETRUCCI, L. J. T. ; ZANELATO, E. B. ; OLIVEIRA, T. F. . Evaluation of the Pozzolanic Activity of Residue From the Paper Industry. The Minerals, Metals \& Materials Series. 15ed.: Springer International Publishing, 2017, v. 2, p. 657-662. 